\title{
The LBTI hunt for observable signatures of terrestrial systems (HOSTS) survey: a key NASA science program on the road to exoplanet imaging missions
}

W. Danchi, V. Bailey, G. Bryden, D. Defrère, C. Haniff, et al.

W. Danchi, V. Bailey, G. Bryden, D. Defrère, C. Haniff, P. Hinz, G. Kennedy, B. Mennesson, R. Millan-Gabet, G. Rieke, A. Roberge, E. Serabyn, A. Skemer, K. Stapelfeldt, A. Weinberger, M. Wyatt, "The LBTI hunt for observable signatures of terrestrial systems (HOSTS) survey: a key NASA science program on the road to exoplanet imaging missions," Proc. SPIE 9146, Optical and Infrared Interferometry IV, 914607 (24 July 2014); doi: 10.1117/12.2056681

Event: SPIE Astronomical Telescopes + Instrumentation, 2014, Montréal, Quebec, Canada 


\title{
The LBTI Hunt for Observable Signatures of Terrestrial Systems (HOSTS) Survey: A key NASA science program on the road to exoplanet imaging missions
}

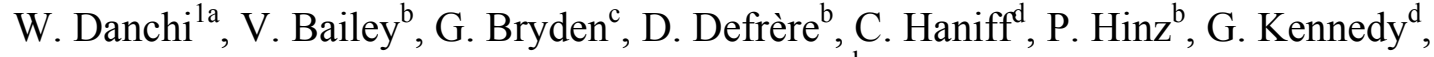 \\ B. Mennesson ${ }^{\mathrm{c}}$, R. Millan-Gabet ${ }^{\mathrm{e}}$, G. Rieke ${ }^{\mathrm{b}}$, A. Roberge ${ }^{\mathrm{a}}$, E. Serabyn ${ }^{\mathrm{c}}$, \\ A. Skemer ${ }^{b}$, K. Stapelfeldt ${ }^{\mathrm{a}}$, A. Weinberger ${ }^{\mathrm{f}}$, M. Wyatt ${ }^{\mathrm{d}}$ \\ ${ }^{\mathrm{a}}$ NASA Goddard Space Flight Center, Greenbelt, MD; ${ }^{\mathrm{b}}$ University of Arizona, Tucson, AZ, \\ ${ }^{\mathrm{c}}$ Jet Propulsion Laboratory, Pasadena, CA; ${ }^{\mathrm{d}}$ University of Cambridge, Cambridge, UK; \\ ${ }^{\mathrm{e}}$ California Institute of Technology, Pasadena, CA; ${ }^{\mathrm{f}}$ Carnegie Institution, Washington, DC
}

\begin{abstract}
The Hunt for Observable Signatures of Terrestrial planetary Systems (HOSTS) program on the Large Binocular Telescope Interferometer (LBTI) will survey nearby stars for faint exozodiacal dust (exozodi). This warm circumstellar dust, analogous to the interplanetary dust found in the vicinity of the Earth in our own system, is produced in comet breakups and asteroid collisions. Emission and/or scattered light from the exozodi will be the major source of astrophysical noise for a future space telescope aimed at direct imaging and spectroscopy of terrestrial planets (exoEarths) around nearby stars. About $20 \%$ of nearby field stars have cold dust coming from planetesimals at large distances from the stars (Eiroa et al. 2013, A\&A, 555, A11; Siercho et al. 2014, ApJ, 785, 33). Much less is known about exozodi; current detection limits for individual stars are at best $\sim 500$ times our solar system's level (aka. 500 zodi). LBTI-HOSTS will be the first survey capable of measuring exozodi at the 10 zodi level $(3 \sigma)$. Detections of warm dust will also reveal new information about planetary system architectures and evolution. We will describe the motivation for the survey and progress on target selection, not only the actual stars likely to be observed by such a mission but also those whose observation will enable sensible extrapolations for stars that will not be observed with LBTI. We briefly describe the detection of the debris disk around $\eta$ Crv, which is the first scientific result from the LBTI coming from the commissioning of the instrument in December 2013, shortly after the first time the fringes were stabilized.
\end{abstract}

Keywords: debris disks, exozodiacal dust, stellar interferometry, nulling interferometry, exoplanet detection, infrared astronomy

\section{INTRODUCTION}

NASA is currently embarked on a long-term mission to search for exoplanets in the habitable zones of nearby sun-like stars, ideally to detect and characterize rocky Earth-sized planets, which might have liquid water and oxygen, and hence evidence for non-equilibrium atmospheric chemistry caused by life.

Over the past two decades mission concepts have been developed at infrared and at visible wavelengths, initially called the Terrestrial Planet Finder - Interferometer (TPF-I) and the Terrestrial Planet Finder - Coronagraph (TPF-C), respectively [1]. The latter mission concept has provided the scientific and technical foundation for current studies of the Wide Field Infrared Space Telescope (WFIRST) - Astrophysics Focused Telescope Asset (AFTA) internal coronagraph, which utilizes one of two 2.4-m visible wavelength space telescopes donated by the intelligence community to NASA. Also, at the present time NASA is studying two Probe-class mission concepts ( $\sim 1 \mathrm{~B}$ USD), one with an internal coronagraph on a 1+ $\mathrm{m}$ class telescope, called Exo-C [2], and another that is a study of a star-shade concept called Exo-S, with a telescope of a similar size and a moderately-sized starshade [3]. These activities stem from the endorsement by Astro2010 Decadal Survey [4] of the search for new worlds. The survey chose the WFIRST mission

\footnotetext{
${ }^{1}$ william.c.danchi@nasa.gov; phone 1301 286-4586; fax 1301 286-7230
} 
as its top priority for large missions, and the New Worlds Technology Development Program, was selected to be the top priority in the medium-size mission category. The probe-class mission concepts are being studied in case the WFIRSTAFTA - coronagraph mission is not brought into the implementation phase.

The search for life on planets around other worlds is observationally extremely difficult for two main reasons. First, at visible wavelengths (at around $600 \mathrm{~nm}$ ) the contrast-ratio between the star and the reflected light for the planet when the planet is at maximum elongation is of the order of $10^{-10}$ and the contrast ratio in the mid-infrared $(10 \mu \mathrm{m})$ is of the order of $10^{-7}$. Second, dusty disks, which are the remnants of the primordial protoplanetary disks in which the planets formed, act as a source of astrophysical background noise and source confusion in the measurements if the dust density is larger than about ten times that of our solar system and/or if the dusty material is clumpy, respectively [5].

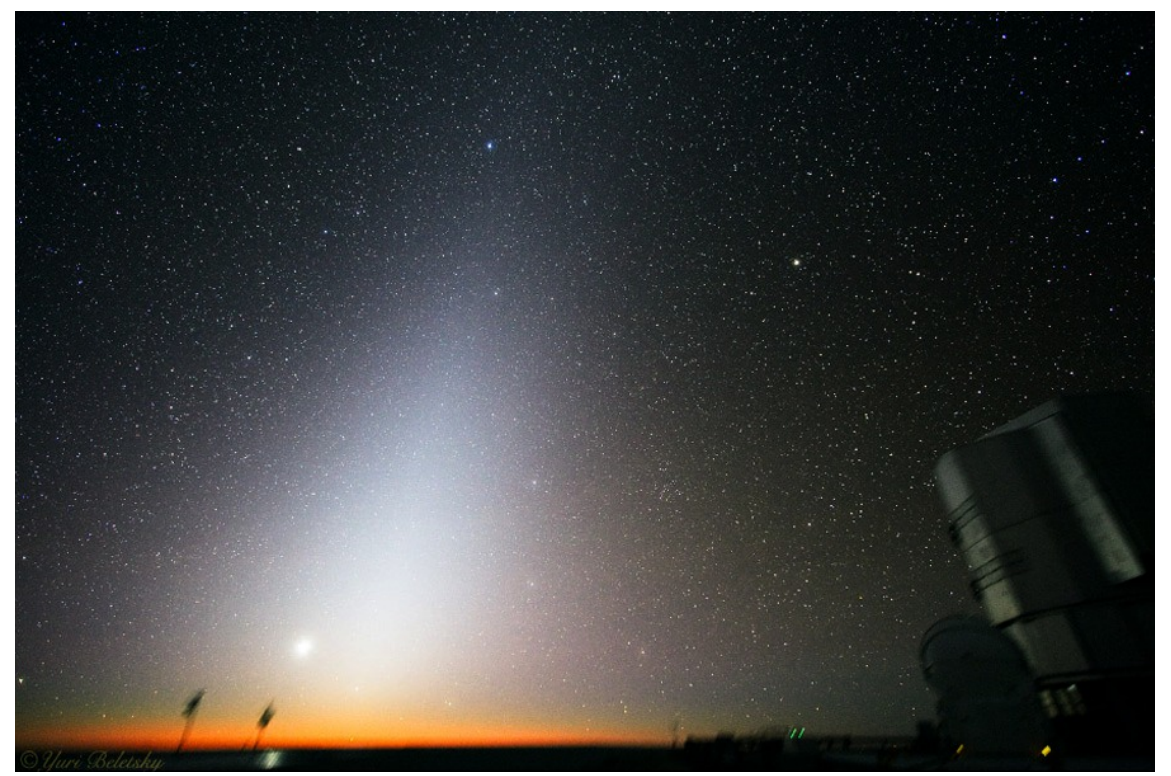

Figure 1. The view of zodiacal dust as seen from the site of the Very Large Telescope (VLT) in Chile. (Courtesy $\mathrm{ESO} / \mathrm{VLT}$ ).

Around our solar system, such dust is created by collisions of asteroids and the evaporation of comets. The scattered sunlight, called zodiacal light, as seen from the site of the Very Large Telescope (VLT) in Chile, is shown in Figure 1. This warm dust extends from the asteroid belt inward to the point at which dust is sublimated by the heat from the Sun. Cold dust also exists in our solar system in the Edgeworth-Kuiper belt. The dust that affects observations of exoplanets in the habitable zone is the warm component of the dust, called exozodiacal dust by analogy with our own zodiacal dust.

The Astro2010 survey committee discussed the importance of determining exozodiacal dust levels around nearby stars and it specifically mentioned the "... need to characterize the level of zodiacal light present so as to determine, in a statistical sense if not for individual prime targets, at what level starlight scattered from dust will hamper planet detection" [4]. The survey committee recommended accelerating work in this area, and if the sensitivity of groundbased surveys could not achieve a level of less than ten times that of the solar system, then a small space mission, such as the EXoplanetary Circumstellar Environment and Dust Explorer (EXCEDE) mission currently under NASA study, may be required in order to determine the characteristics of a large-scale mission for the detection and characterization of Earth twins [4].

The effect of the exozodiacal dust on the performance of planet finding and characterizations missions has been discussed thoroughly in a paper by Roberge et al. [5]. Figure 2 displays the integration time to detect a planet as a function of zodiacal dust levels (or zodi level) for coronagraphic instruments on telescopes of different diameters - 2- , 4-, and 8-meters. In this figure, the integration time goes as the telescope diameter, $\mathrm{D}^{-4}$, and linearly with the zodi level. Here we use the term "zodi" to mean that the detected emission is equivalent to that of our own solar system, using the 
optical depth and vertical and in plane density and temperature structure of the debris disk as defined by the Kelsall model (Kelsall et al. [6]).

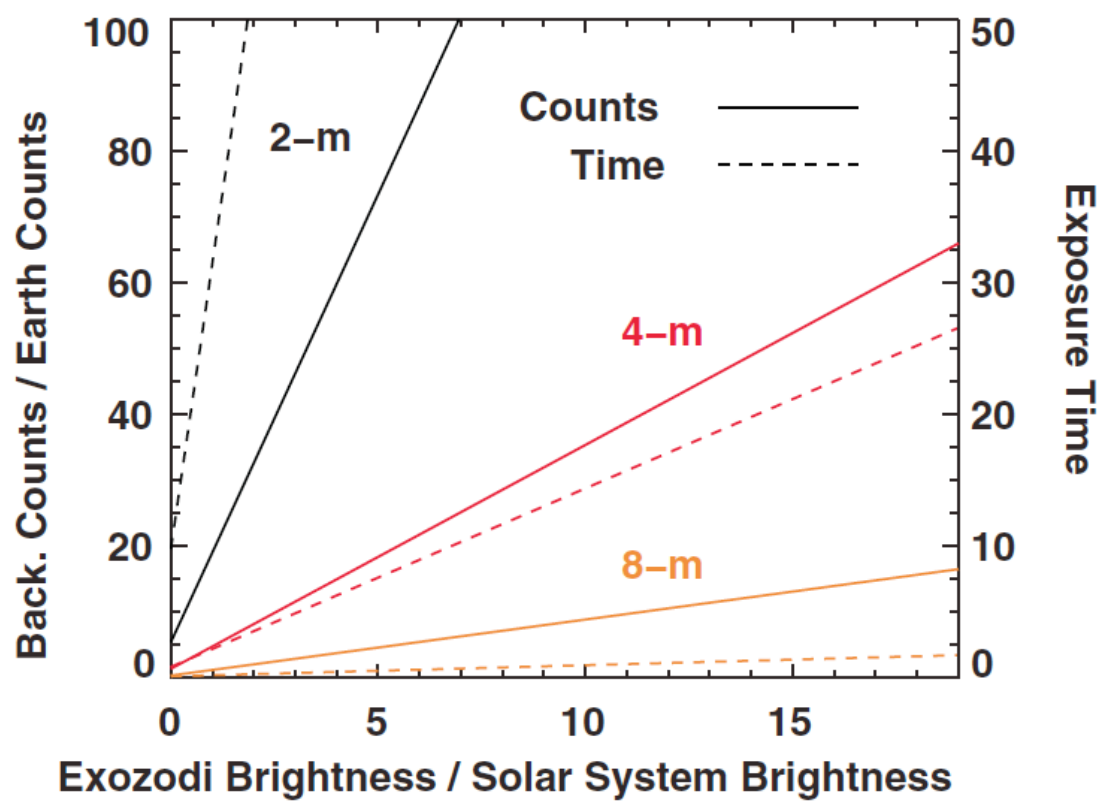

Figure 2. Background Counts divided by Earth Counts as a function of exozodi brightness divided by the solar system zodi brightness. Solid lines denote counts and dashed lines denote exposure time, for a 2-m (black), 4-m (red), and 8-m (orange) diameter telescopes with a coronagraph, operating at visible wavelengths. The exposure time is in arbitrary units for a given signal-to-noise ratio. (Adapted from Roberge et al. [4]).

This paper describes the ground-based exozodiacal dust survey being conducted by NASA with the Large Binocular Telescope Interferometer (LBTI), called the Hunt for Observable Signatures of Terrestrial Planets (HOSTS) program. The personnel in the program consists of the LBTI instrument team led by Principal Investigator, P. Hinz, with instrument scientists, D. Defrère and A. Skemer, all from the University of Arizona, and a science team competitively selected by NASA, composed of scientists from a number of institutions. This paper is one of several contributions by the team for this conference, with a discussion of aspects of co-phasing the LBTI in a contribution led by Defrère et al. later in this session (9146-9) [7], commissioning of the LBTI as a nulling interferometer and imager by Hinz et al. on Wednesday (9146-28) [8], and improvements to the accuracy of null depth measurements by Defrère et al. on Friday (9146-48) [9].

For the remainder of the paper we discuss the exozodiacal dust problem, current observational limits, and why the LBTI was chosen for this survey in Section 2, the HOSTS survey team, charter, and sample selection in Section 3, and status and some early scientific results obtained during the commissioning process in Section 4.

\section{THE EXOZODIAL DUST PROBLEM}

\subsection{Limitations of Existing Measurements}

Over the last two+ decades a succession of cooled infrared satellites -- IRAS, ISO, Spitzer and Herschel -- have allowed astronomers to search for infrared excesses in the Spectral Energy Distributions (SEDs) of main-sequence stars in the solar neighborhood. Using the $24 \mu \mathrm{m}$ channel of the MIPS photometer on Spitzer, Rieke et al. [10], [11] performed a study of 266 main-sequence A stars within a distance of $150 \mathrm{pc}$ of the Sun. They showed that the fraction of stars with an excess (as compared to that of the emission from the photosphere) decreased from $\sim 25 \%$ for the youngest stars in the sample ( 10 Myr) to $\sim 1 \%$ for stars older than $>190$ Myr. Strong excesses were observed well past the initial falloff ( $\sim 500 \mathrm{Myr}$ ) indicating that dust was being created in episodic collisions of asteroids or other small bodies within these systems. 
Other investigations have focused on nearby F, G, K stars. Bryden et al. [12] summarized results at 24 and $70 \mu \mathrm{m}$ on a sample of 146 stars with known planets. It was found that 14 of 146 stars had infrared excesses at 24 and/or $70 \mu \mathrm{m}$, with just a single star with an excess only at $24 \mu \mathrm{m}$. The remaining 13 stars had stronger emission at $70 \mu \mathrm{m}$ consistent with cold dust $>10 \mathrm{AU}$ from the star. These authors found a marginally lower detection rate for stars with planets than those without planets, but the result was inconclusive as the observed difference was not statistically significant. Lawler et al. (2009) used the Infrared Spectrometer (IRS) instrument on a sample of 152 nearby solar type stars. Data from the 30-34 $\mu \mathrm{m}$ band of IRS gave a fraction of stars with an excess that was about $12 \%$, similar to that of Bryden et al. [12]. The fraction at the short wavelength band $(8.5-12 \mu \mathrm{m})$ was only $1 \%$. These observations constrained the mean zodiacal dust level to be less than 1000 times that of the solar system for warm dust, and 100 times that of the solar system for cool dust. No correlation was seen with metallicity or spectral type, as noted by Bryden et al. [13], but all of the studies were consistent with an age correlation, i.e., stars less than $1 \mathrm{Gyr}$ old were more likely to have excesses than older stars like the Sun.

At $24 \mu \mathrm{m}$ with Spitzer, detections required fluxes more than $10 \%$ above the photosphere due to errors in estimation of the photospheric levels, as well as flat fielding errors (see Siercho et al. [14]; Bryden et al. [13]). At $70 \mu \mathrm{m}$ the Spitzer observations were limited by extragalactic background source confusion and by Galactic cirrus (Bryden et al. [13]).

The Herschel Space Observatory had sufficient sensitivity (with the PACS instrument at 100 and $160 \mu \mathrm{m}$ ) to detect the emission from the equivalent of our own Edgeworth-Kuiper belt around nearby solar type stars. Two Open Time Key Projects were devoted to debris disk observations. The DUNES project (C. Eiroa, PI) focused on a volume-limited sample of 133 FGK stars within $25 \mathrm{pc}$ of the Sun. The key feature of DUNES was that for each star the integration time is adjusted to detect its photosphere with $\mathrm{SNR}=5$. The DEBRIS project (P.I., B. Mathews) observed a larger number of stars with the integration time adjusted to a particular flux density limit. Both projects shared 106 stars. The DUNES project increased the incidence rate of discs around the DUNES sample from $\sim 12.1 \%+/-5 \%$ before Herschel to $20.2 \%$ $+/-2 \%$, and $\sim 52 \%$ of detected disks were resolved (Eiroa et al. [15]).

There has been relatively little observational work on the warm component of debris disks, corresponding to dust in the habitable zone. Nulling interferometry has become an established technique to observe this component of debris disks. With nulling interferometry an achromatic $\pi$ phase shift is introduced between the electric fields coming from two telescopes before they are interfered, producing a broad-band dark fringe at the location of the host star, and bright fringes $\lambda /(2 \mathrm{~B})$ from the dark fringe where the interferometer is most sensitive to thermal emission from dust or a planet, where $\lambda$ is the wavelength of observation and $\mathrm{B}$ is the interferometer baseline. This technique was invented by Bracewell [16], but it took many years before it was employed in exozodi observations with the Keck Interferometer Nuller and the BLINC instrument at the MMT as discussed below. Nulling interferometry is at the heart of the TPF-I mission concept, and progress in this field up to the end of the last decade is described in the chapter on infrared detection of exoplanets in the Exoplanet Community Report [1].

The nulling instruments measure a quantity called the leakage, which is zero only for a perfect instrument with a pointlike star. There is a minimum leakage due to instrumental errors such as residual wavefront and path-length errors, as well as the finite size of the host star. The leakage is the difference between the null signal from a target star minus the null signal from a reference star. The leakage can also be related to traditional interferometric quantities such as the visibility, where the null quantity, $N$, is given by $N=(1-V) /(1+V)$, where $V$ is the magnitude of the complex visibility as it is usually defined, e.g., the Fourier transform of the brightness distribution of the source (see papers by Barry et al. [17], Serabyn et al. [18] for more detailed discussions).

Two studies focused on determining the mean level around nearby main-sequence stars. The first was published by Liu et al. [19] who used the BLINC-MIRAC instrument on the MMT, which had its AO system, the MMTAO, installed at that time. This study focused on six nearby stars: four A stars, one F, and one K star. No warm dust emission was detected from any of the objects, and the 3- $\sigma$ upper limits ranged from 220 to 10,000 zodis. The second study was the Keck Interferometer Nuller (KIN) survey of 25 stars at $\mathrm{N}$ band (Millan-Gabet et al. [20]). There were 3 detections ( $\eta$ Crv, $\gamma$ Oph, and $\alpha \mathrm{Aql})$, and 22 non-detections. The non-detections were consistent with a 3- $\sigma$ mean zodi level less than 150 zodis, if all the stars were considered to be a single class of objects. For the KIN observations, the largest source of error was not the formal error due to the scatter in the calibrated leakages, but instead it was the night-to-night variation of the calibrated data on the same stars, which was called the "external" error (Millan-Gabet et al. [20], and Colavita et al. [21], [22]). 


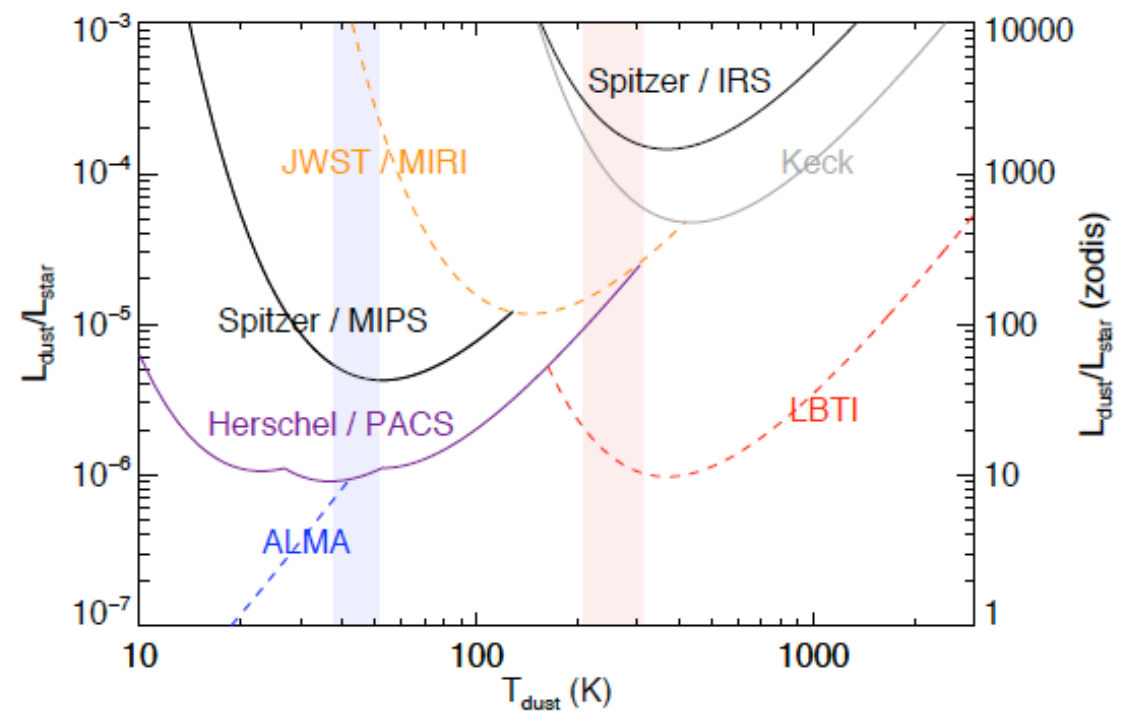

Figure 3. Comparison of the sensitivity of major instruments as a function of dust temperature, using $\mathrm{L}_{\text {dust }} / \mathrm{L}_{\text {star }}$ as a measure of sensitivity, where $\mathrm{L}_{\text {dust }}$ is the luminosity of dust and $\mathrm{L}_{\text {star }}$ is the luminosity of a star. Note that LBTI has the best sensitivity of all existing and near-term instruments for dust between 150 and $1000 \mathrm{~K}$, including the MIRI instrument on JWST. (Adapted from Roberge et al. [4]).

\subsection{Instrumental Sensitivity Comparisons and LBTI's Advantages}

The LBTI has emerged as the best current instrument for improved detection limits for warm debris disks and exozodi studies around nearby stars. Figure 3 displays the results of a comparison of the sensitivity of instruments used for exozodi studies (Roberge et al. [5]), using a simple model of performance for each instrument, described briefly here. Assuming that the stellar spectrum is a described by a Rayleigh-Jeans law and the dust emission is a single temperature blackbody, the fraction luminosity of dust compared to the host $\operatorname{star}\left(L_{d u s t} / L *\right)$ can expressed as:

$$
\frac{L_{\text {dust }}}{L_{*}}=\left(\frac{F_{\text {dust }}}{F_{*}}\right) \frac{k T_{d}^{4}\left(e^{h v / k T_{d}}-1\right)}{h v T_{*}^{3}},
$$

where $F_{d u s t}$ and $F_{*}$, are dust and stellar fluxes at a frequency $v$, the frequency of the observation, and $T_{*}$ is the effective temperature of the star (see Bryden et al. [13]). If $F_{\text {dust }} / F_{*}$ is replaced by 3 times the one-sigma $(1-\sigma)$ uncertainty of the flux of the dust compared to that of the star, $\sigma_{F_{\text {dust }} / F_{*}}$ and plotted as a function of $T_{d u s t}$, the result is a set of sensitivity curves for a set of instruments (described in detail in Roberge et al. [5]). Each curve has a minimum where the blackbody emission from dust peaks at the center frequency of the instrument. In the figure note that the range of dust temperatures in the Kuiper belt that corresponds to radial distances of 30-55 AU is shown by the light blue stripe, and the temperature range of dust in the habitable zone is shown by the pink stripe, corresponding to radial distances of 0.81.8 AU, assuming a star like the Sun.

The outstanding performance of the LBTI as shown in Figure 3 results from a number of unique instrumental features. The LBTI has only three warm reflections before the cold optics of the Universal Beam Combiner (UBC) as well as adaptive secondaries with high-order wavefront correction that provides very high Strehl ratios at $\mathrm{N}$ band (>98\%). The UBC feeds the components of the LBTI, consisting of NOMIC, the mid-infrared camera and nuller, as well as LMIRCAM, an additional camera operating at $\mathrm{L}$ and $\mathrm{M}$ bands. The throughput (transmission) to the detector is $\sim 20 \%$ and the emissivity is very low, $<10 \%$, giving it the highest photometric sensitivity of any ground-based interferometer operating in the mid-infrared. The expected photometric uncertainty is $0.01 \%$ compared to $0.3 \%$ for the Keck Interferometer Nuller (KIN). 
Besides the high photometric sensitivity, the angular resolution of the LBTI is well-suited to observations of warm debris disks. The first bright fringe is located at an angular distance of $\lambda /(2 \mathrm{~B})$ from the central dark fringe, where $\lambda$ is the wavelength of observation and B is the baseline. For the case of LBTI at $10 \mu \mathrm{m}$ and with a center-to-center spacing of $14.4 \mathrm{~m}$ for the two 8.4-m diameter mirrors, the first bright fringe is located at 72 milliarcsec (mas), close to the angular size of $1 \mathrm{AU}$ at $10 \mathrm{pc}$, which is 100 mas, corresponding to warm dust in the habitable zone at an earth distance from a star like the Sun.

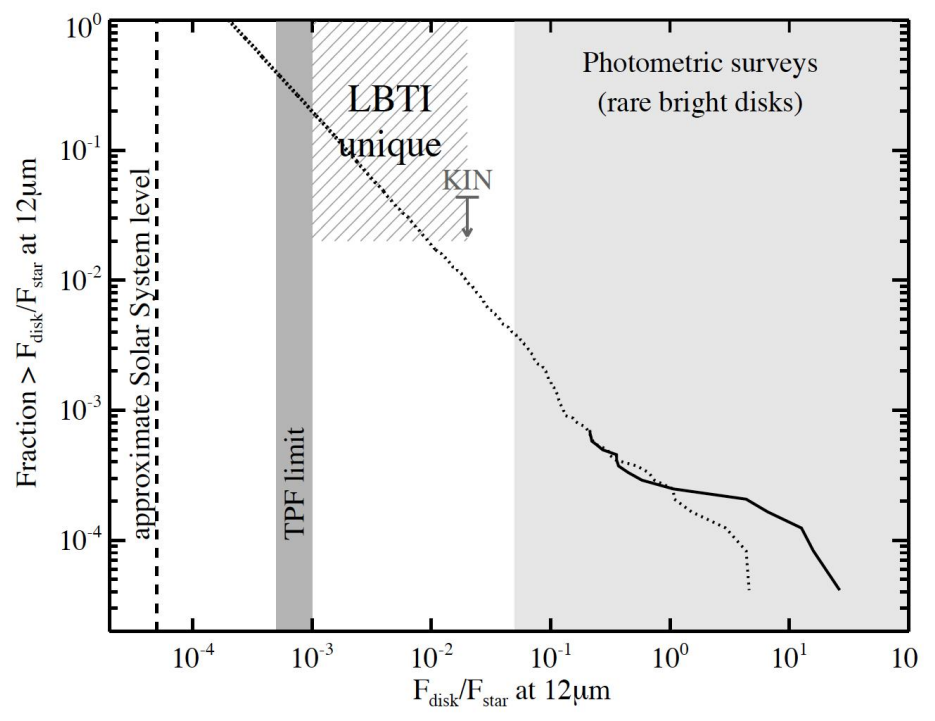

Figure 4. Luminosity function for stars between 0 and $13 \mathrm{Gyr}$ old. The solid line denotes the observed distribution function based on photometric surveys and the dotted line is an extrapolated luminosity function assuming an in situ collisional model consistent with the observational data. The limit from the KIN survey is shown and the unique space occupied by the LBTI survey is shown by the cross-hatched region on the plot. (Kennedy et al. [22]).

The NOMIC camera itself includes the nuller (NIL) at $\mathrm{N}$ band and PhaseCam, which operates at $\mathrm{H}$ and $\mathrm{K}$ bands to stabilize the null within NIL. NOMIC includes a cold field stop with two slits, a pupil wheel with dual and single apertures of varying sizes, dual 12-hole non-redundant aperture masks, and dual spider-annuli masks. The filter wheels include a variety of set of narrow band filters $(0.7 \mu \mathrm{m}$ wide centered at $7.9,8.9,9.1,9.8,11.9,12.5 \mu \mathrm{m})$, wide filters $\left(\mathrm{N}^{\prime}\right.$ -- 9.9-12.4 $\mu \mathrm{m}$, also 8-13.8 $\mu \mathrm{m}, 8.1-9.4 \mu \mathrm{m}, 10.1-11.1 \mu \mathrm{m}), 18 \mu \mathrm{m}$ and $24 \mu \mathrm{m}$ filters, and a grism for low-resolution spectroscopy. Further details of the characteristics and current performance of the LBTI can be found in the contributions to this conference by Hinz et al. [8] and Defrère et al. [7] as mentioned previously.

The importance of LBTI to the study of exozodiacal dust can be seen in Figure 4, which displays an extrapolated luminosity function for exozodiacal dust disks (Kennedy et al. [23]). In this figure the dark line displays the observed luminosity function, which exists only for the dusty stars observed in the photometric surveys discussed previously. An extrapolated luminosity function, assuming an in situ collisional model consistent with the observational data, is shown by the dotted line. The LBTI uniquely explores the luminosity function at the low fractional luminosity levels. Limits for TPF and an upper limit derived from the KIN survey are also displayed in this figure.

\section{THE LBTI HOSTS PROGRAM}

\subsection{LBTI Hosts Team}

The HOSTS team was competitively selected by NASA to support a 60-night survey of at least 50 nearby solar-type stars to determine individual zodi levels or upper limits for those stars and the mean zodi level for these stars taken as a single population, such as was done in previous work with the Keck Interferometer Nuller. The key questions the team will answer from the survey are: 
1. What is the exozodi luminosity function for nearby stars?

2. Does the level of cold/hot dust correlate with exozodi level?

3. How does the exozodi level vary with stellar spectral type?

The HOST team is the science team for the LBTI and its membership and skill set complements those of the instrument team located at the University of Arizona. The team first met in September 2012 and consists of G. Bryden with collaborator F. Morales (both at JPL) , W. Danchi (GSFC), B. Mennesson with collaborator G. Serabyn (both at JPL), K. Stapelfeldt (GSFC), A. Weinberger (Carnegie Institution) with collaborator A. Roberge (GSFC), and M. Wyatt with collaborators C. Haniff, G. Kennedy, O. Panic, and A. Shannon (Cambridge University, UK). During the past year and a half the team has been focused on defining the target sample, modeling, supporting commissioning observations, the data pipeline, and data analysis methods. Although each team member has a specialty, anyone can contribute to any activity as needed or desired. The LBTI is currently being commissioned and the survey is expected to begin after the Operational Readiness Review (ORR) in early 2015 and will last until approximately the 2017-2018 time frame.

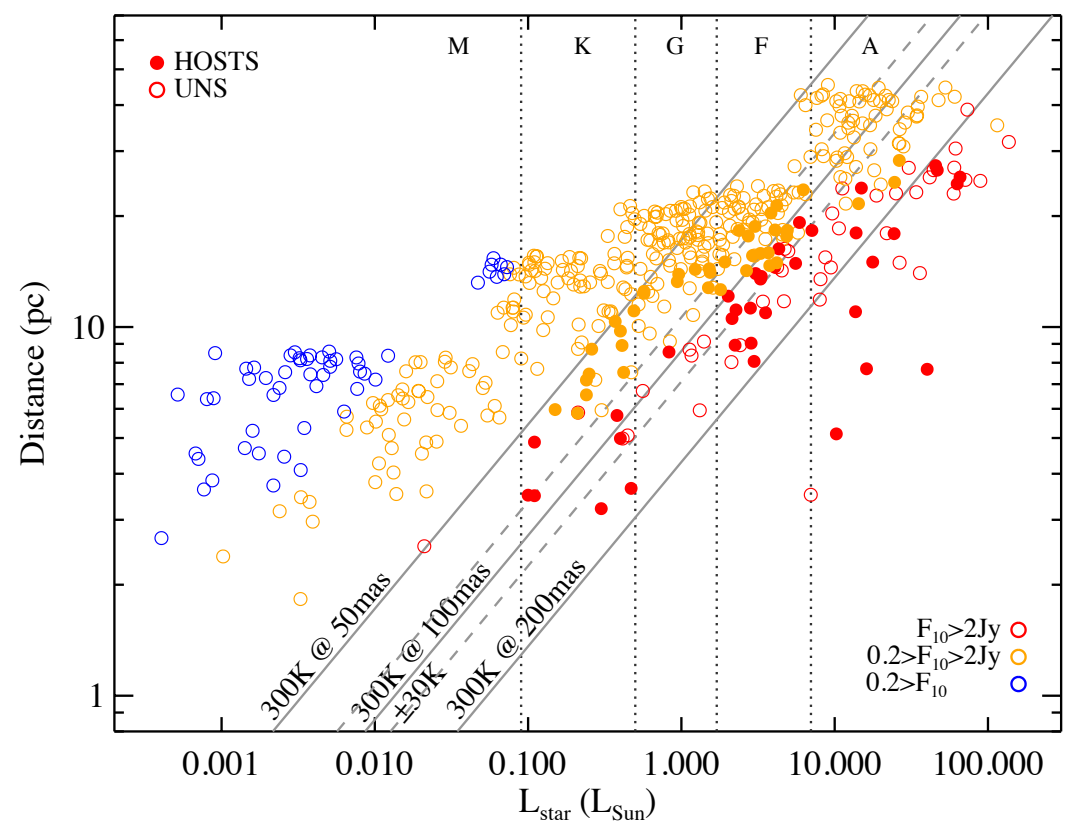

Figure 5. The distance of stars from Sun sample as a function of luminosity, grouped according to $10-\mu \mathrm{m}$ flux. The open circles represent the UNS sample, and the red and orange closed circles represent the HOSTS sample. The solid lines denote distance as a function of luminosity given a blackbody at a temperature of $300 \mathrm{~K}$ at angular scales of 50,100 , and 200 mas, and the dashed lines $+/-30 \mathrm{~K}$ from the $300 \mathrm{~K}$ line. Dotted lines are approximate boundaries in luminosity for the spectral classes (Weinberger et al. [24]).

\subsection{Target Selection}

The team has worked diligently to define the target sample. A paper is in preparation describing the target selection methodology and results (Weinberger et al. [24]). Two sample types have been determined based on three different methods. For FGK stars, which are of greatest interest for exoplanet direct imaging missions, two differing selection methods were compared, one we call the Mission Oriented Selection is determined by applying a target selection code derived from work on the New Worlds Observer (Turnbull et al. [25]), and the other is a Mission Independent Selection derived from the Unbiased Nearby Survey (UNS) sample (Phillips et al. [26]). Both selection methods were applied to stars with spectral types later than F5 and resulted in a sample of 49 nearby sun-like stars, which we call the "Sun-like Sample". A second sample is called the "Sensitivity Sample", and it consists of stars earlier than F4 for which the LBTI has the best sensitivity. This sample consists of 26 stars of spectral type A0-F4, 12 of which have known excesses. This 
sample will allow us to probe the exozodi luminosity function at the lowest levels, allowing for a potential extrapolation to the luminosity function of FGK stars in the Sun-like Sample, and it also allows us to correlate cold dust from those stars in this sample with known excesses with detections of warm dust from LBTI, which can give us greater insight into the dynamics and evolution of exozodiacal dust. The main exclusions for the two samples were binaries with separation less than 1.5 arcsec and all the stars had to have declination greater than -30 degrees. Figure 5 displays the distance of stars from Sun as a function of luminosity, grouped according to $10-\mu \mathrm{m}$ flux, with red open circles are stars with $10-\mu \mathrm{m}$ flux greater than $2 \mathrm{Jy}$, which were part of the UNS sample, orange open circles are stars from 0.2 to $2 \mathrm{Jy}$, and the blue open circles are stars with fluxes less than $0.2 \mathrm{Jy}$. The red and orange closed circles represent stars from the HOSTS sample. The solid lines denote distance as a function of luminosity given a blackbody at a temperature of $300 \mathrm{~K}$ at angular scales of 50,100, and 200 mas, and the dashed lines $+/-30 \mathrm{~K}$ from the $30 \mathrm{~K}$ line. Note the best stars in the sample are essentially the closest FGK stars (Weinberger et al. [24]).

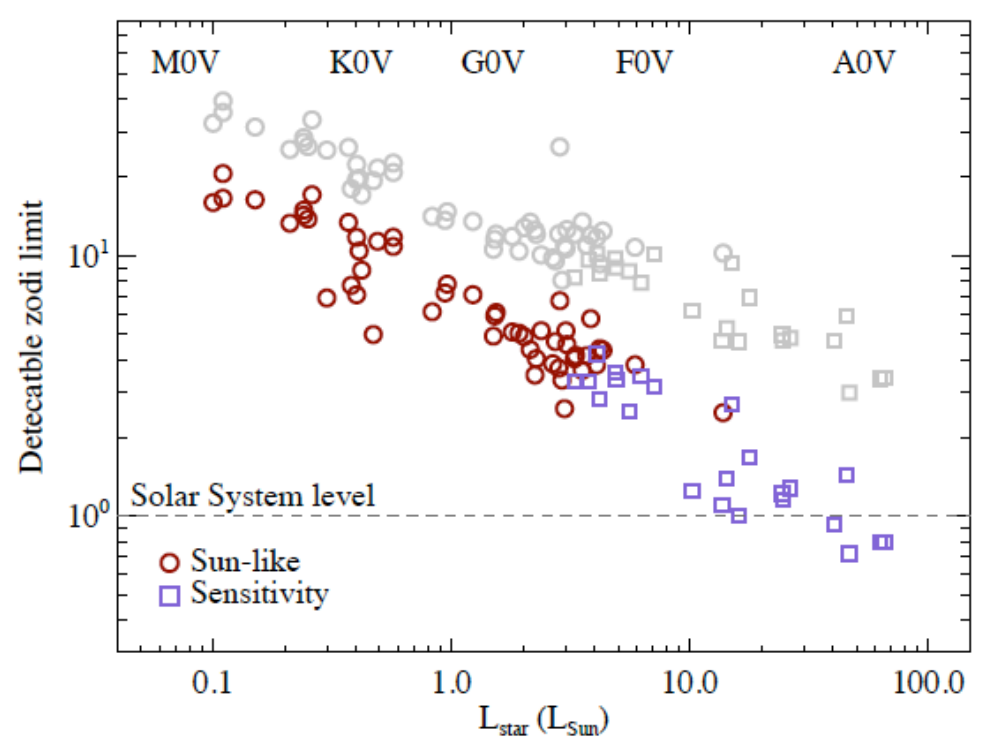

Figure 6. Prediction of LBTI sensitivity limits in terms of solar system zodi units as a function of stellar luminosity assuming the LBTI reaches a stabilized null depth of $10^{-4}$. Stars from the Sun-like sample are denoted by the red open circles and the Sensitivity sample by the blue open squares. The zodi level for the Solar System is indicated by the dashed line. The gray symbols indicate limits for a pessimistic narrow-disk scenario where the disks cover the habitable zone only from 320 to $210 \mathrm{~K}$ (Kennedy et al. [27]).

\subsection{Modeling}

A significant effort has been undertaken by our team to create a modeling framework for the LBTI observations in order to determine dust density levels from detections and to place upper limits on the dust levels for non-detections. A paper is currently in preparation describing this effort [27]. The LBTI observations of thermal dust emission can then be converted into predictions and upper limits for scattered light observations. In this way the LBTI observations will help optimize the target list for individual stars and as a class for visible wavelength direct imaging missions such WFIRSTAFTA, Exo-C, Exo-S, and ATLAST that are currently in a study phase. The modeling effort connects simple physical models of dust disks with the well-known modeling program zodipic [28], which utilizes the Kelsall model [6] of our own zodiacal dust. The modeling effort also allows for predictions of the sensitivity of LBTI for the two target samples discussed in the previous section. Figure 6 displays such a prediction assuming the LBTI reaches a stabilized null depth of $10^{-4}$, with the Sun-like sample shown in the red open circles and the Sensitivity sample shown in the blue open squares. Note that the bulk of the stars in the Sun-like sample from K0 to F0 have detectable zodi limits from about 10 to 3 zodies, while the Sensitivity sample stars are clustered near the Solar System zodi level and even lower for the most luminous stars A stars in the sample. This figure clearly shows that the LBTI has the potential to determine the 
zodi levels of nearby stars, both individually and as a class, necessary to formulate the direct imaging missions in support of the Astro2010 Decadal Survey goals.

\section{STATUS AND EARLY RESULTS FROM COMMISSIONING PERIOD}

\subsection{Commissioning}

Commissioning activities for the LBTI continue and are discussed in detail in the paper by Hinz et al. [8] in this conference. The first fringes were obtained by the LBTI in October 2010, but without the adaptive secondaries. First null measurements were obtained in September 2012, and the first stabilized null measurements were obtained in the observing run in December 2013. Figure 7 displays the results obtained in December 2013 when the PhaseCam loop was closed for the first time. The left panel displays a histogram of a sequence of null measurements before the phasing loop was closed. In this plot, note that the number of measurements of the null depth range from near zero to $100 \%$, which is a consequence of the random variation of the path length, and hence phase between the two telescopes. The right panel is the histogram of the nulling sequence with the phase loop closed. In this plot the histogram clearly shows most of the measurements peaking close to a null depth of a few percent, consistent with the residual rms phase variation. Note that the shape of the histogram in the right panel is consistent with expectations from statistics given the amount of residual phase noise, and can be fitted using a statistical technique based on Bayesian methods developed by Hanot et al. [29], and Mennesson et al. [30], to better determine the null depth and the null precision than can be done with simple descriptive statistics.
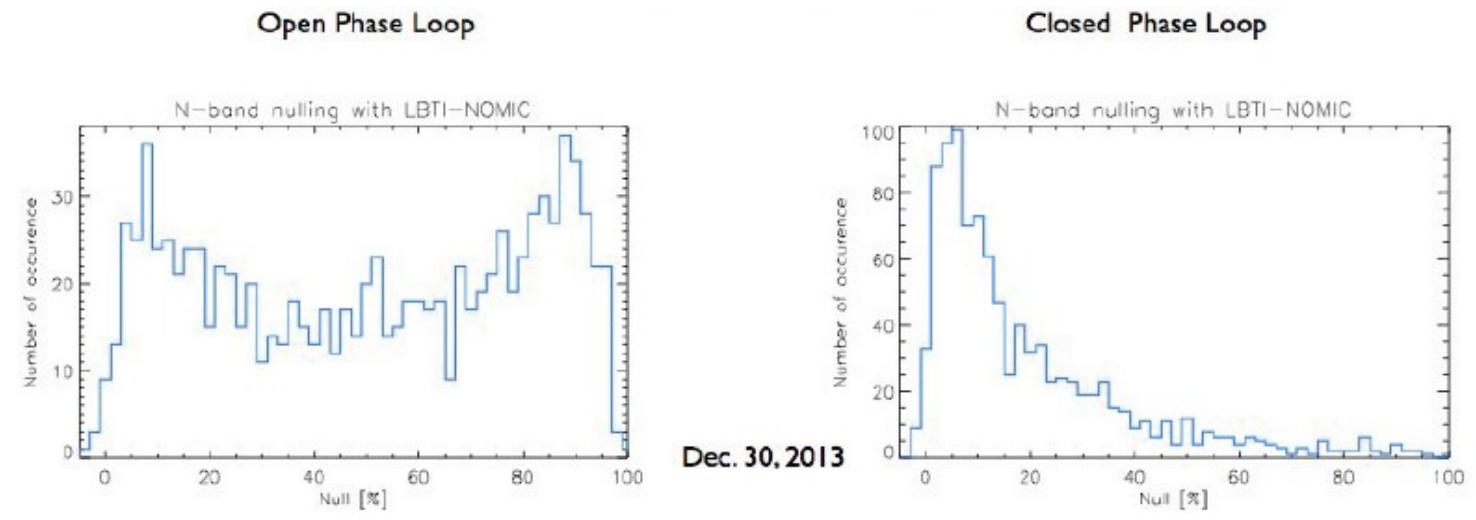

Figure 7. Results obtained in December 2013 when the PhaseCam loop was closed for the first time. The panel displays a histogram of a sequence of null measurements before the phasing loop was closed. In this plot, note that the number of measurements of the null depth range from near zero to $100 \%$, which is a consequence of the random variation of the path length, and hence phase, between the two telescopes. The right panel is the histogram of the nulling sequence with the phase loop closed. In this plot the histogram clearly shows most of the measurements peaking close to a null depth of a few percent, consistent with the residual rms phase variation.

\subsection{First science results: $\eta$ Corvi}

Our first science results were obtained during a commissioning run in February 12, 2014, with the star $\eta$ Crv. Three hours of nulling data were taken near transit. Figure 8 displays the region $(\mathrm{u}, \mathrm{v})$ space for the observations, along with an image of the object taken by the Herschel PACS instrument at $70 \mu \mathrm{m}$. The orientation of the baseline was close to the direction of the major axis of the outer disk observed by Herschel. The Herschel observations were consistent with a disk have an inclination angle of 46.8 degrees and a position angle of 116.3 degrees (Duchene et al. [31]). Figure 9 displays null leakage measurements from the LBTI as a function of observation time in UT hours for the best $5 \%$ of the data as defined by measurements with smallest nulls in each set. The red symbols denote the measurements of $\eta$ Crv and the blue symbols display measurements of the calibrator stars. The black line is an average of the calibrator measurements. The dotted lines are the average of the error in the null measurements for the calibrators. This is a clear detection of the warm disk around $\eta$ Crv, which had previously been detected with the Spitzer IRS instrument with an excess of $17 \%$ [32], and an excess of $4 \%$ observed with the Keck Interferometer Nuller (Millan-Gabet et al. [20]). These results are currently being analyzed in detail and will be published in an upcoming paper by Defrère et al. [33]. 


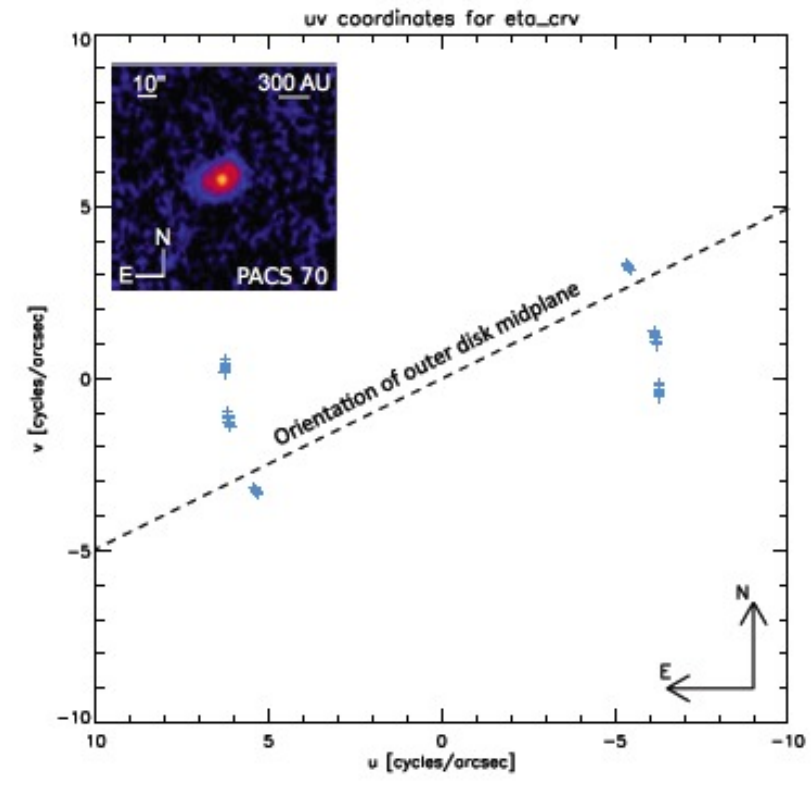

Figure 8. The region in $(\mathrm{u}, \mathrm{v})$ space for the observations, along with an image of the object taken by the Herschel PACS instrument at $70 \mu \mathrm{m}$. The orientation of the baseline was close to the direction of the major axis of the outer disk observed by Herschel. The Herschel observations were consistent with a disk have an inclination angle of 46.8 degrees and a position angle of 116.3 degrees (Duchene et al. [31]).

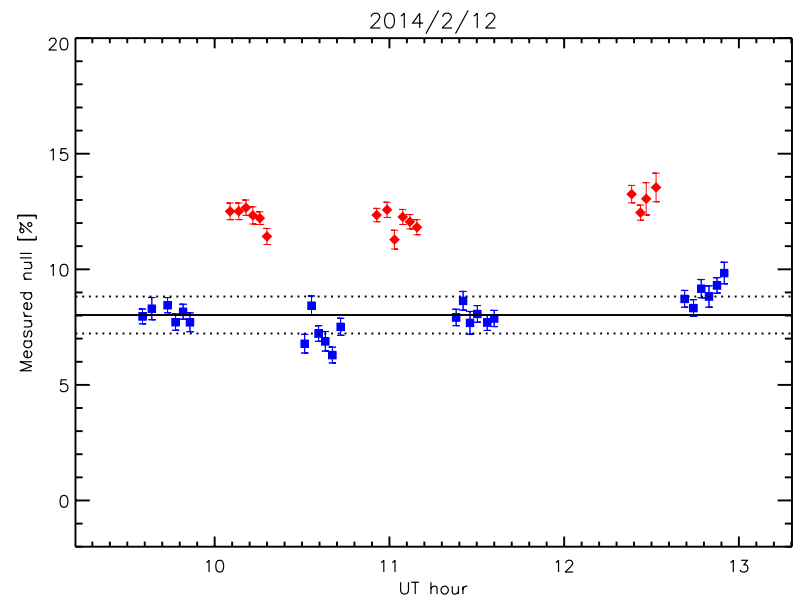

Figure 9. Null measurements for $\eta \mathrm{Crv}$ in which only the best $5 \%$ of the measurements are averaged. The red symbols denote the measurements of $\eta \mathrm{Crv}$ and the blue symbols are measuremes of the calibrator stars. The black line is an average of the calibrator measurements, and the average error in the null measurements for the calibrators is indicated by the dotted lines. The excess is detected in the $\mathrm{N}^{\prime}$ band $(9.81-12.41 \mu \mathrm{m})$ over a field-of-view of $\sim 140$ mas in radius, corresponding to $\sim 2.6 \mathrm{AU}$ at the distance of $\eta \mathrm{Crv}$. 


\section{SUMMARY AND CONCLUSIONS}

The LBTI HOSTS project will for the first time measure exozodi levels around nearby direct imaging sun-like target stars at the level of 10 times or lower than that of the solar system zodiacal dust level. Two types of target samples have been selected. One is called the "Sun-like" sample and it consists of 49 stars with spectral types later than F5; the other is called the "Sensitivity" sample and it consists of 26 stars having spectral types earlier than F4. For the latter sample, dust levels of the order of one solar system zodi or lower can be expected for some of the stars having the earliest spectral types. The LBTI HOSTS project is currently in a commissioning phase, and null fringes have been stabilized using PhaseCam. Improvements to the fringe stability are expected as improvements to the phasing camera software control loops are underway. The first detection of warm zodiacal dust has been achieved during commissioning with a clear detection of dust around $\eta$ Crv. Papers discussing some of the technical aspects of LBTI have been presented at this conference. Papers are in preparation for submission to refereed journals regarding the target selection, modeling, and first results on $\eta \mathrm{Crv}$ as indicated in the text. Next year, after commissioning is completed and the HOSTS project passes the Operational Readiness Review, then the formal survey will begin and will be completed in the next several years.

\section{ACKNOWLEDGMENTS}

The research described in this paper is supported by the Astrophysics Division of the National Aeronautics and Space Administration and the Large Binocular Telescope Observatory, and by a grant from the European Union through ERC grant number 279973 (GMK \& MCW).

\section{REFERENCES}

[1] Lawson, P. R., Traub, W. A., and Unwin, S. C. (editors), "Exoplanet Community Report," JPL Publication 09-3, Chapters 2 and 4 (2009).

[2] Stapelfeldt, K. et al, Exo-C interim report (2014).

[3] Seager, S. et al., Exo-S interim report (2014).

[4] Blandford, R. et al., "New Worlds, New Horizons," Astro2010 Decadal Survey, National Academies Press, Washington, DC (2010).

[5] Roberge, A. et al., PASP, 124, 799 (2012).

[6] Kelsall, T. et al., ApJ, 508, 44 (1998).

[7] Defrère, D. et al., paper 9146-9, this conference (2014).

[8] Hinz, P. et al., paper 9146-28, this conference (2014).

[9] Defrère, D. et al., paper 9146-48, this conference, talk only, no written contribution (2014).

[10] Rieke, G. et al., ApJS, 154, 25 (2004).

[11] Rieke, G. et al., ApJ, 620, 1020 (2005).

[12] Bryden, G. et al., ApJ, 705, 1206 (2009).

[13] Bryden, G. et al., ApJ, 636, 1098 (2006).

[14] Siercho, J. M. et al., ApJ, 785, 33 (2014).

[15] Eiroa, C. et al., A\&A, 555, A11 (2013).

[16] Bracewell, R., Nature, 274, 780 (1978).

[17] Barry, R. K. et al., ApJ, 677, 1253 (2008).

[18] Serabyn, E. et al., ApJ, 748, 55 (2012).

[19] Liu, W. et al., ApJ, 693, 1500 (2009).

[20] Millan-Gabet, R. et al., ApJ, 734, 67 (2011).

[21] Colavita, M. et al., PASP, 121, 1120 (2009).

[22] Colavita, M. et al., PASP, 122, 795 (2010).

[23] Kennedy, G. \& Wyatt, M. MNRAS, 433, 2334 (2013).

[24] Weinberger, A. et al., in prep. (2014).

[25] Turnbull, M. et al., PASP, 124, 418 (2012).

[26] Phillips, N. et al. MNRAS, 403, 1089 (2010).

[27] Kennedy, G. et al., in prep. (2014).

[28] Kuchner, M., freely available software package. 
[29] Hanot, C. et al., ApJ, 729, 110 (2011).

[30] Mennesson, B. et al., ApJ, 736, 14 (2011).

[31] Duchene et al. ApJ, 784, 148 (2014).

[32] Lisse, C. et al., ApJ, 747, 93 (2012).

[33] Defrère, D. et al., in prep. (2014).

Proc. of SPIE Vol. $9146914607-12$

Downloaded From: https://www.spiedigitallibrary.org/conference-proceedings-of-spie on 7/11/2018 Terms of Use: https://www.spiedigitallibrary.org/terms-of-use 